\title{
Satisfiability Modulo Abstraction for Separation Logic with Linked Lists
}

\author{
Aditya Thakur ${ }^{1}$, Jason Breck ${ }^{1}$, and Thomas Reps ${ }^{1,2}$ \\ 1 University of Wisconsin; Madison, WI, USA \{adi, jbreck, reps\}@cs.wisc.edu \\ 2 GrammaTech, Inc.; Ithaca, NY, USA
}

\begin{abstract}
Separation logic is an expressive logic for reasoning about heap structures in programs. This paper presents a semi-decision procedure for deciding unsatisfiability of formulas in a fragment of separation logic that includes predicates describing points-to assertions $(x \mapsto y)$, acyclic-list-segment assertions $(\mathbf{l} \mathbf{s}(x, y))$, logical-and, logical-or, separating conjunction, and septraction (the DeMorgan-dual of separating implication). The fragment that we consider allows negation at leaves, and includes formulas that lie outside other separation-logic fragments considered in the literature.

The semi-decision procedure is designed using concepts from abstract interpretation. The procedure uses an abstract domain of shape graphs to represent a set of heap structures, and computes an abstraction that over-approximates the set of satisfying models of a given formula. If the over-approximation is empty, then the formula is unsatisfiable.

We have implemented the method, and evaluated it on a set of formulas taken from the literature. The implementation is able to establish the unsatisfiability of formulas that cannot be handled by other existing approaches.
\end{abstract}

\section{Introduction}

Separation logic [29] is an expressive logic for reasoning about heap-allocated data structures in programs. It provides a mechanism for concisely describing program states by explicitly localizing facts that hold in separate regions of the heap. In particular, a "separating conjunction" $\left(\varphi_{1} * \varphi_{2}\right)$ asserts that the heap can be split into two disjoint regions ("heaplets") in which $\varphi_{1}$ and $\varphi_{2}$ hold, respectively [29]. A "septraction" $\left(\varphi_{1}-\circledast \varphi_{2}\right)$ asserts that a heaplet $h$ can be extended by a disjoint heaplet $h_{1}$ in which $\varphi_{1}$ holds, to create a heaplet $h_{1} \cup h$ in which $\varphi_{2}$ holds [34]. The $-\circledast$ operator is sometimes called existential magic wand, because it is the DeMorgan-dual of the magic-wand operator "-*" (also called separating implication); i.e., $\varphi_{1}-\circledast \varphi_{2}$ iff $\neg\left(\varphi_{1}-* \neg \varphi_{2}\right)$.

The use of separation logic in manual, semi-automated, and automated verification tools is a burgeoning field $[3,12,24,13,17]$. Most of these incorporate some form of automated reasoning for separation logic, but only limited fragments of separation logic are typically handled.

This paper presents a semi-decision procedure for deciding the unsatisfiability of formulas in a fragment of separation logic that includes predicates describing 
points-to assertions $(x \mapsto y)$, acyclic-list-segment assertions $(\mathbf{l s}(x, y))$, emptyheap assertions (emp), and their negations; separating conjunction; septraction; logical-and; and logical-or. The fragment considered only allows negation at the leaves of a formula ( $\$ 2.1)$, but still contains formulas that lie outside of previously considered fragments $[2,27,26,22,19]$. The semi-decision procedure can prove validity of implications of the form

$$
\psi \Rightarrow\left(\varphi_{i} \wedge \bigwedge_{j} \psi_{j}-* \varphi_{j}\right)
$$

where $\varphi_{i}$ and $\varphi_{j}$ are formulas that contain only $\wedge, \vee$, and positive or negative occurrences of emp, points-to, or ls assertions; and $\psi$ and $\psi_{j}$ are arbitrary formulas in the logic fragment defined in $\S 2.1$. Consequently, we believe that ours is the first procedure that can prove the validity of formulas that contain both ls and the magic-wand operator $-*$. Furthermore, the semi-decision procedure is able to prove unsatisfiability of interesting classes of formulas that are outside of previously considered fragments, including (i) formulas that use conjunctions of separating-conjunctions with $\boldsymbol{l s}$, such as

$$
(\mathbf{l s}(a 1, a 2) * \mathbf{l} \mathbf{s}(a 2, a 3)) \wedge(\neg \mathbf{e m p} * \neg \mathbf{e m p}) \wedge(a 1 \mapsto e 1 * \text { true }) \wedge e 1=\text { nil },
$$

and (ii) formulas that contain both ls and septraction $(-\circledast)$, such as

$$
(a 3 \mapsto a 4-\circledast \mathbf{l s}(a 1, a 4)) \wedge(a 3=a 4 \vee \neg \mathbf{l} \mathbf{s}(a 1, a 3)) .
$$

The former are useful for describing overlapped data structures; the latter are useful in dealing with interference effects when using rely/guarantee reasoning to verify programs with fine-grained concurrency $[34,7]$.

The key insight behind our approach is that the semi-decision procedure is designed using concepts from abstract interpretation [10]. Given a formula $\varphi$, the semi-decision procedure sets up an appropriate abstract domain that is tailored for representing information about the meanings of subformulas of $\varphi$. In particular, it uses an abstract domain of shape graphs [30] to represent a set of heap structures. The proof calculus that we present computes an abstraction that over-approximates the set of satisfying models of the given formula. If the over-approximation is empty, then the formula is unsatisfiable. If the formula is satisfiable, then the procedure reports a set of abstract models.

This use of abstract domains to prove unsatisfiability places our work squarely in a recent line of research on using abstract values drawn from an abstract domain as a way to represent knowledge in implementations of decision procedures $[14,33,32,15,16]$ (a technique we call "Satisfiability Modulo Abstraction"). Our work is the first to apply this idea to a fragment of separation logic.

The nature of our semi-decision procedure is thus much different from other decision procedures for fragments of separation logic that we are aware of. Most previous decision procedures are proof-theoretic. In some sense, our method is model-theoretic: it uses explicitly instantiated sets of 3 -valued structures to represent overapproximations of the models of subformulas.

Contributions. The contributions of the paper include the following: 
- We show how a canonical-abstraction domain can be used to overapproximate the set of heaps that satisfy a separation-logic formula $(\S 2)$.

- We present rules for calculating the overapproximation of a separation-logic formula for a fragment of separation logic that consists of separating conjunction, septraction, logical-and, and logical-or (§4).

- The semi-decision procedure is parameterized by a shape abstraction, and can be instantiated to handle (positive or negative) literals for points-to or acyclic-list-segment assertions - and hence can prove the validity of implications of the kind shown in formula (1) ( 44$)$.

$\S 3$ illustrates the key concepts used in the semi-decision procedure. We evaluated our approach on a set of formulas taken from the literature ( $\$ 5)$. To the best of our knowledge, the implementation is able to establish the unsatisfiability of formulas that cannot be handled by other existing approaches.

\section{Separation Logic and Canonical Abstraction}

In this section, we provide background on separation logic and introduce the separation-logic fragment considered in the paper. We then show how a canonical-abstraction domain can be used to approximate the set of models that satisfy a separation-logic formula.

\subsection{Syntax and Semantics of Separation Logic}

Formulas in our fragment of separation logic (SL) are defined as follows:

$$
\begin{aligned}
\varphi: & :=\varphi \wedge \varphi|\varphi \vee \varphi| \varphi * \varphi|\varphi-\circledast \varphi| \text { atom } \mid \neg \text { atom } \\
\text { atom }: & =\text { true }|\operatorname{emp}| x=y|x \mapsto y| \operatorname{ls}(x, y)
\end{aligned}
$$

The set of literals, denoted by lits, is the union of the positive and negative atoms of SL.

The semantics of SL is defined with respect to memory "statelets", which consist of a store and a heaplet. A store is a function from variables to values; a heaplet is a finite function from locations to locations. Let Loc and Var be disjoint countably infinite sets, neither of which contain nil.

$$
\begin{aligned}
& \text { Val } \stackrel{\text { def }}{=} \operatorname{Loc} \uplus\{\mathrm{nil}\} \quad \text { Store } \stackrel{\text { def }}{=} \mathrm{Var} \rightarrow \mathrm{Val} \\
& \text { Heaplet } \stackrel{\text { def }}{=} \text { Loc } \rightarrow_{\text {fin }} \text { Val Statelet } \stackrel{\text { def }}{=} \text { Store } \times \text { Heaplet }
\end{aligned}
$$

Loc represents heap-node addresses. The domain of $h$, $\operatorname{dom}(h)$, represents the set of addresses of cells in the heaplet. Two heaplets $h_{1}, h_{2}$ are disjoint, denoted by $h_{1} \# h_{2}$, if $\operatorname{dom}\left(h_{1}\right) \cap \operatorname{dom}\left(h_{2}\right)=\emptyset$. Given two disjoint heaplets $h_{1}$ and $h_{2}$, $h_{1} \cdot h_{2}$ denotes their disjoint union $h_{1} \uplus h_{2}$. A statelet is denoted by a pair $(s, h)$.

Satisfaction of an SL formula $\varphi$ with respect to statelet $(s, h)$ is defined in Fig. 1. $\llbracket \varphi \rrbracket$ denotes the set of statelets that satisfy $\varphi: \llbracket \varphi \rrbracket \stackrel{\text { def }}{=}\{(s, h) \mid(s, h) \models \varphi\}$.

\section{$2.2 \quad 2$-Valued Logical Structures}

We model full states - not statelets-by 2-valued logical structures. A logical structure provides an interpretation of a vocabulary $\operatorname{Voc}=\left\{e q, p_{1}, \ldots, p_{n}\right\}$ of predicate symbols (with given arities). $\operatorname{Voc}_{k}$ denotes the set of $k$-ary symbols. 


$$
\begin{aligned}
& (s, h) \models \varphi_{1} \wedge \varphi_{2} \quad \text { iff } \quad(s, h) \models \varphi_{1} \text { and }(s, h) \models \varphi_{2} \\
& (s, h) \models \varphi_{1} \vee \varphi_{2} \quad \text { iff } \quad(s, h) \models \varphi_{1} \text { or }(s, h) \models \varphi_{2} \\
& (s, h) \models \varphi_{1} * \varphi_{2} \quad \text { iff } \exists h_{1}, h_{2} . h_{1} \# h_{2} \text { and } h_{1} \cdot h_{2}=h \text { and }\left(s, h_{1}\right) \models \varphi_{1} \text { and }\left(s, h_{2}\right) \models \varphi_{2} \\
& (s, h) \models \varphi_{1}-\circledast \varphi_{2} \text { iff } \exists h_{1} . h_{1} \# h \text { and }\left(s, h_{1}\right) \models \varphi_{1} \text { and }\left(s, h_{1} \cdot h\right) \models \varphi_{2} \\
& (s, h) \models \neg \text { atom } \quad \text { iff }(s, h) \not \models \text { atom } \\
& (s, h) \models \text { true } \quad \text { iff true } \\
& (s, h) \models \mathbf{e m p} \quad \text { iff } \operatorname{dom}(h)=\emptyset \\
& (s, h) \models x=y \quad \text { iff } s(x)=s(y) \\
& (s, h) \models x \mapsto y \quad \text { iff } \operatorname{dom}(h)=\{s(x)\} \text { and } h(s(x))=s(y) \\
& (s, h) \models \mathbf{l} \mathbf{s}(x, y) \quad \text { iff if } s(x)=s(y) \text { then } \operatorname{dom}(h)=\emptyset \text {, } \\
& \text { else there is a nonempty acyclic path from } s(x) \text { to } s(y) \text { in } h \text {, } \\
& \text { and this path contains all heap cells in } h
\end{aligned}
$$

Fig. 1: Satisfaction of an SL formula $\varphi$ with respect to statelet $(s, h)$.

\begin{tabular}{||l|l||}
\hline Predicate & Intended Meaning \\
\hline$e q\left(v_{1}, v_{2}\right)$ & Do $v_{1}$ and $v_{2}$ denote the same memory cell? \\
$q(v)$ & Does pointer variable q point to memory cell $v ?$ \\
$n\left(v_{1}, v_{2}\right)$ & Does the $n$-field of $v_{1}$ point to $v_{2} ?$ \\
\hline
\end{tabular}

Fig. 2: Core predicates used when representing states made up of acyclic linked lists.

Definition 1. A 2-valued logical structure $S$ over $\operatorname{Voc}$ is a pair $S=\langle U, \iota\rangle$, where $U$ is the set of individuals, and $\iota$ is the interpretation. Let $\mathbb{B}=\{0,1\}$ be the domain of truth values. For $p \in \operatorname{Voc}_{i}, \iota(p): U^{i} \rightarrow \mathbb{B}$. We assume that $e q \in V o c_{2}$ is the identity relation: (i) for all $u \in U, \iota(e q)(u, u)=1$, and (ii) for all $u_{1}, u_{2} \in U$ such that $u_{1}$ and $u_{2}$ are distinct individuals, $\iota(e q)\left(u_{1}, u_{2}\right)=0$.

The set of 2-valued logical structures over Voc is denoted by 2-STRUCT[Voc].

A concrete state is modeled by a 2-valued logical structure over a fixed vocabulary $\mathcal{C}$ of core predicates. Core predicates are part of the underlying semantics of the linked structures that make up the states of interest. Fig. 2 lists the core predicates that are used when representing states made up of acyclic linked lists.

Without loss of generality, vocabularies exclude constant and function symbols. Constant symbols can be encoded via unary predicates, and $n$-ary functions via $n+1$-ary predicates. In both cases, we need integrity rules-i.e., global constraints that restrict the set of structures considered to the ones that we intend. For instance, we use a subset of the unary predicates, denoted by $P \operatorname{Var} \subseteq \operatorname{Voc}_{1}$, to encode pointer variables (i.e., $x \in P$ Var encodes program variable $\mathrm{x}$ ), and binary predicate $n \in \mathrm{Voc}_{2}$ to encode list-node linkages. In essence, the following integrity rules restrict each $x \in P$ Var to serve as a (possibly undefined) constant, and restrict relation $n$ to encode a partial function:

$$
\begin{aligned}
& \text { for each } x \in P \operatorname{Var}, \forall v_{1}, v_{2}: x\left(v_{1}\right) \wedge x\left(v_{2}\right) \Rightarrow e q\left(v_{1}, v_{2}\right) \\
& \forall v_{1}, v_{2}, v_{3}: n\left(v_{3}, v_{1}\right) \wedge n\left(v_{3}, v_{2}\right) \Rightarrow e q\left(v_{1}, v_{2}\right)
\end{aligned}
$$




\subsection{Connecting 2-Valued Logical Structures and SL Statelets}

We use unary domain predicates, typically denoted by $d, d^{\prime}, d_{1}, \ldots, d_{k} \in \mathrm{Voc}_{1}$, to pick out regions of the heap that are of interest in the state that a logical structure models. The connection between 2-valued logical structures and SL statelets is formalized by means of the operation $\left.S\right|_{(d, \cdot)}$, which performs a projection of structure $S$ with respect to a domain predicate $d$ :

$$
\begin{array}{r}
\left.S\right|_{(d, \cdot)} \stackrel{\text { def }}{=}(s, h), \text { where } s=\left(\begin{array}{c}
\left\{(p, u) \mid p \in P \operatorname{Var}^{S}, u \in U^{S} \text {, and } p(u)\right\} \\
\cup\left\{(q, \operatorname{nil}) \mid q \in P \operatorname{Var}^{S} \text { and } \neg \exists v: q(v)\right\}
\end{array}\right) \\
\text { and } h=\left\{\left(u_{1}, u_{2}\right) \mid u_{1}, u_{2} \in U^{S}, d\left(u_{1}\right) \text {, and } n\left(u_{1}, u_{2}\right)\right\} .
\end{array}
$$

The subscript " $(d, \cdot)$ " serves as a reminder that in Eqn. (3), only $u_{1}$ needs to be in the region defined by $d$. We lift the projection operation to apply to a set SS of 2-valued logical structures as follows: $\left.\mathrm{SS}\right|_{(d, \cdot)} \stackrel{\text { def }}{=}\left\{\left.S\right|_{(d, \cdot)} \mid S \in \mathrm{SS}\right\}$.

\subsection{Representing Sets of SL Statelets using Canonical Abstraction}

In the framework of Sagiv et al. [30] for logic-based abstract-interpretation, 3valued logical structures provide a way to overapproximate possibly infinite sets of 2-valued structures in a finite way that can be represented in a computer. The application of Eqns. (2) and (3) to 3-valued structures means that the abstract-interpretation machinery developed by Sagiv et al. provides a finite way to overapproximate a possibly infinite set of SL statelets.

In 3 -valued logic, a third truth value, denoted by $1 / 2$, represents uncertainty. The set $\mathbb{T} \stackrel{\text { def }}{=} \mathbb{B} \cup\{1 / 2\}$ of 3 -valued truth values is partially ordered " $l \sqsubset 1 / 2$ for $l \in \mathbb{B}$ ". The values 0 and 1 are definite values; $1 / 2$ is an indefinite value.

Definition 2. A 3-valued logical structure $S=\langle U, \iota\rangle$ is almost identical to a 2-valued structure, except that $\iota$ maps each $p \in V_{\text {Voc }}$ to a 3-valued function $\iota(p): U^{i} \rightarrow \mathbb{T}$. In addition, (i) for all $u \in U, \iota(e q)(u, u) \sqsupseteq 1$, and (ii) for all $u_{1}, u_{2} \in U$ such that $u_{1}$ and $u_{2}$ are distinct individuals, $\iota(e q)\left(u_{1}, u_{2}\right)=0$. (An individual $u$ for which $\iota(e q)(u, u)=1 / 2$ is called a summary individual.)

The set of 3-valued logical structures over Voc is denoted by 3-STRUCT[Voc]. Note that 2-STRUCT[Voc $\subsetneq$ 3-STRUCT[Voc $]$.

As we will see below, a summary individual may represent more than one individual from certain 2-valued structures.

A 3-valued structure can be depicted as a directed graph with individuals as graph nodes (see Fig. 3). A summary individual is depicted with a doubleruled border. A unary predicate $p \in P$ Var is represented in the graph by having an arrow from the predicate name $p$ to all nodes of individuals $u$ for which $\iota(p)(u) \sqsupseteq 1$. An arrow between two nodes indicates that a binary predicate holds for the corresponding pair of individuals. A predicate value of $1 / 2$ is indicated by a dotted arrow, a value of 1 by a solid arrow, and a value of 0 by the absence of an arrow. A unary predicate $p \in\left(\mathrm{Voc}_{1}-P\right.$ Var $)$ is listed, with its value, inside the 
node of each individual $u$ for which $\iota(p)(u) \sqsupseteq 1$. A nullary predicate is displayed in a rectangular box.

To define a suitable abstraction of 2-valued logical structures, we start with the notion of structure embedding [30]:

Definition 3. Given $S=\langle U, \iota\rangle$ and $S^{\prime}=\left\langle U^{\prime}, \iota^{\prime}\right\rangle$, two 3-valued structures over the same vocabulary Voc, and $f: U \rightarrow U^{\prime}$, a surjective function, $f$ embeds $S$ in $S^{\prime}$, denoted by $S \sqsubseteq^{f} S^{\prime}$, if for all $p \in$ Voc and $u_{1}, \ldots, u_{k} \in U$,

$$
\iota(p)\left(u_{1}, \ldots, u_{k}\right) \sqsubseteq \iota^{\prime}(p)\left(f\left(u_{1}\right), \ldots, f\left(u_{k}\right)\right)
$$

If, in addition,

$$
\iota^{\prime}(p)\left(u_{1}^{\prime}, \ldots, u_{k}^{\prime}\right)=\bigsqcup_{u_{1}, \ldots, u_{k} \in U, \text { s.t.f }\left(u_{i}\right)=u_{i}^{\prime}, 1 \leq i \leq k} \iota(p)\left(u_{1}, \ldots, u_{k}\right)
$$

then $S^{\prime}$ is the tight embedding of $S$ with respect to $f$, denoted by $S^{\prime}=f(S)$. (Note that we overload $f$ to also mean the mapping on structures $f:$ 3-STRUCT[Voc] $\rightarrow$ 3-STRUCT[Voc] induced by $f: U \rightarrow U^{\prime}$.)

Intuitively, $f(S)$ is obtained by merging individuals of $S$ and by defining the valuation of predicates accordingly (in the most precise way). The relation $\sqsubseteq^{\text {id }}$, which will be denoted by $\sqsubseteq$, is the natural information order between structures that share the same universe. One has $S \sqsubseteq^{f} S^{\prime} \Leftrightarrow f(S) \sqsubseteq^{\text {id }} S^{\prime}$.

However, embedding alone is not enough. The challenge for representing and manipulating sets of 2-valued structures is that the universe of a structure is of a priori unbounded size. Consequently, we need a method that, for a 2 -valued structure $\langle U, \iota\rangle \in 2$-STRUCT[Voc], abstracts $U$ to an abstract universe $U^{\sharp}$ of bounded size. Intuitively, the solution involves (i) identifying an a priori bounded number of abstract individuals $U^{\sharp}$, (ii) defining a surjective function $f: U \rightarrow U^{\sharp}$, and (ii) performing a tight embedding of $S$ with respect to $f$. Given $U^{\sharp}$ and $f$, we can define the following Galois connection:

$$
\begin{array}{cr}
\wp(2-\mathrm{STRUCT}[\mathrm{Voc}]) & \stackrel{\gamma_{f}}{\leftrightarrows} \text { 3-STRUCT[Voc] } \\
\alpha_{f}(X)=\bigsqcup_{S \in X} f(S) & \gamma_{f}\left(S^{\sharp}\right)=\left\{S \mid S \sqsubseteq^{f} S^{\sharp}\right\}
\end{array}
$$

In this abstraction, sets of valuations for predicate symbols $\iota:$ Voc $\rightarrow$ $\left(\bigcup_{k} U^{k} \rightarrow \mathbb{B}\right)$ are abstracted with a single abstract valuation $\iota:$ Voc $\rightarrow$ $\left(\bigcup_{k}\left(U^{\sharp}\right)^{k} \rightarrow \mathbb{T}\right)$.

The idea behind canonical abstraction $[30, \S 4.3]$ is to choose a subset $\mathbb{A} \subseteq$ Voc $_{1}$ of abstraction predicates, and to define an equivalence relation $\simeq_{\mathbb{A}^{S}}$ on $U$ that is parameterized by the logical structure $S=\langle U, \iota\rangle \in 2$-STRUCT[Voc] to be abstracted:

$$
u_{1} \simeq_{\mathbb{A}^{S}} u_{2} \Leftrightarrow \forall p \in \mathbb{A}: \iota(p)\left(u_{1}\right)=\iota(p)\left(u_{2}\right) .
$$

This equivalence relation defines the surjective function $f_{\mathbb{A}}^{S}: U \rightarrow\left(U / \simeq_{\mathbb{A}^{S}}\right)$, which maps an individual to its equivalence class. We thus have the Galois connection

$$
\begin{aligned}
& \wp(2-\mathrm{STRUCT}[\mathrm{Voc}]) \underset{\alpha}{\stackrel{\gamma}{\leftrightarrows}} \wp(3 \text {-STRUCT[Voc] }) \\
& \alpha(X)=\left\{f_{\mathbb{A}}^{S}(S) \mid S \in X\right\} \quad \gamma(Y)=\left\{S \mid S^{\sharp} \in Y \wedge S \sqsubseteq^{f} S^{\sharp}\right\},
\end{aligned}
$$


where $f_{\AA}^{S}$ in the definition of $\alpha$ denotes the tight-embedding function for logical structures induced by the node-embedding function $f_{\mathbb{A}}^{S}: U \rightarrow\left(U / \simeq_{\mathbb{A}^{S}}\right)$. The abstraction function $\alpha$ is referred to as canonical abstraction. Note that there is an upper bound on the size of each structure $\left\langle U^{\sharp}, \iota^{\sharp}\right\rangle \in 3$-STRUCT[Voc] that is in the image of $\alpha$ : $\left|U^{\sharp}\right| \leq 2^{|\mathbb{A}|}$ - and thus the power-set of the image of $\alpha$ is a finite sublattice of $\wp(3-\mathrm{STRUCT}[\mathrm{Voc}])$.

For technical reasons, it turns out to be convenient to work with 3 -valued structures other than the ones in the image of $\alpha$; however, we still want to restrict ourselves to a finite sublattice of $\wp(3-\mathrm{STRUCT}[\mathrm{Voc}])$. With this motivation, we make the following definition:

Definition 4. A 3-valued structure $\left\langle U^{\sharp}, \iota^{\sharp}\right\rangle \in 3-S T R U C T[$ Voc $]$ is bounded (with respect to abstraction predicates $\mathbb{A}$ ) if for every $u_{1}, u_{2} \in U^{\sharp}$, where $u_{1} \neq u_{2}$, there exists an abstraction predicate symbol $p \in \mathbb{A} \subseteq$ Voc $c_{1}$ such that $\iota^{\sharp}(p)\left(u_{1}\right)=0$ and $\iota^{\sharp}(p)\left(u_{2}\right)=1$, or $\iota^{\sharp}(p)\left(u_{1}\right)=1$ and $\iota^{\sharp}(p)\left(u_{2}\right)=0$. B-STRUCT[Voc, $\left.\mathbb{A}\right] d e$ notes the set of such structures.

Defn. 4 also imposes an upper bound on the size of a structure $\left\langle U^{\sharp}, \iota^{\sharp}\right\rangle \in$ B-STRUCT $[$ Voc, $\mathbb{A}]$ - again, $\left|U^{\sharp}\right| \leq 2^{|\mathbb{A}|}$ —and thus $\wp(\mathrm{B}$-STRUCT[Voc, $\left.\mathbb{A}]\right)$ is a finite sublattice of $\wp(3$-STRUCT[Voc] $)$. It defines the abstract domain that we use, the abstract domain whose elements are subsets of $B-S T R U C T[V o c, \mathbb{A}]$, which will be denoted by $\mathcal{A}[\mathrm{Voc}, \mathbb{A}]$. (For brevity, we call such a domain a "canonical-abstraction domain", and denote it by $\mathcal{A}$ when Voc and $\mathbb{A}$ are understood.) The Galois connection we work with is thus

$$
\begin{array}{cc}
\wp(2-\mathrm{STRUCT}[\mathrm{Voc}]) & \stackrel{\gamma}{\leftrightarrows} \wp(\text { B-STRUCT }[\text { Voc }, \mathbb{A}])=\mathcal{A}[\text { Voc, } \mathbb{A}] \\
\alpha(X)=\left\{f_{\mathbb{A}}^{S}(S) \mid S \in X\right\} & \gamma(Y)=\left\{S \mid S^{\sharp} \in Y \wedge S \sqsubseteq^{f} S^{\sharp}\right\} .
\end{array}
$$

\section{Overview}

In this section, we illustrate the concepts that we use in the semi-decision procedure using a formula that is unsatisfiable over acyclic linked lists: $x \mapsto y * y \mapsto x$. App. A illustrates the procedure using a formula that is satisfiable over acyclic linked lists: $x \mapsto y-\circledast \mathbf{l} \mathbf{s}(x, z)$.

Consider $\varphi \stackrel{\text { def }}{=} x \mapsto y * y \mapsto x$. We want to compute $A \in \mathcal{A}$ such that $\left.\gamma(A)\right|_{(d, \cdot)} \supseteq \llbracket \varphi \rrbracket$. The key to handling the $*$ operator is to introduce two new domain predicates $d_{1}$ and $d_{2}$, which are used to demarcate the heaplets that must satisfy $\varphi_{1} \stackrel{\text { def }}{=} x \mapsto y$ and $\varphi_{2} \stackrel{\text { def }}{=} y \mapsto x$, respectively. We have designed $\mathcal{A}$ so that there exist $A_{1}, A_{2} \in \mathcal{A}$ such that $\left.\gamma\left(A_{1}\right)\right|_{\left(d_{1}, \cdot\right)}=\llbracket x \mapsto y \rrbracket$ and $\left.\gamma\left(A_{2}\right)\right|_{\left(d_{2}, \cdot\right)}=$ $\llbracket y \mapsto x \rrbracket$, respectively. Tab. 1 describes the abstraction predicates we use. $A_{1}$ and $A_{2}$ each consist of a single 3-valued structure, shown in Fig. 3(b) and Fig. 3(c), respectively. Furthermore, to satisfy $\varphi_{1} * \varphi_{2}, d_{1}$ and $d_{2}$ are required to be disjoint regions whose union is $d$. $\mathcal{A}$ also contains an abstract value, which we will call $D$, that represents this disjointness constraint exactly. $D$ consists of four 3 -valued structures. Fig. 3(a) shows the "most general" of them: it represents two disjoint regions, $d_{1}$ and $d_{2}$, that partition the $d$ region (where each of $d_{1}$ and $d_{2}$ contain 


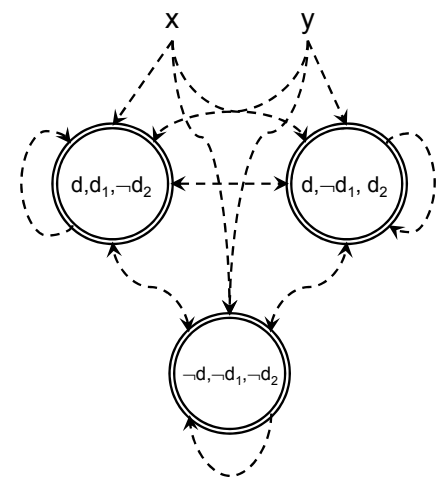

(a)

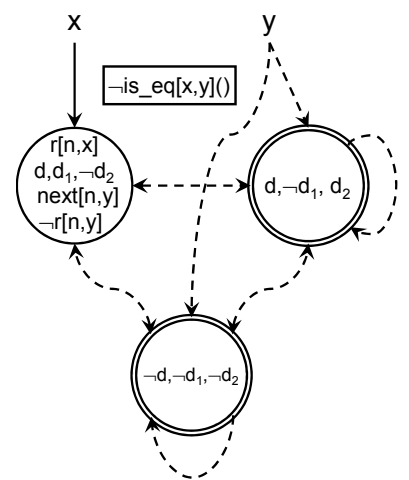

(d)

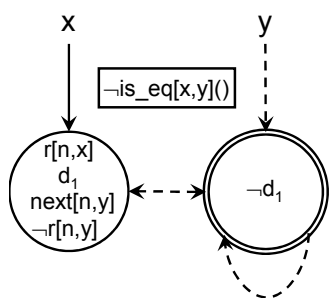

(b)

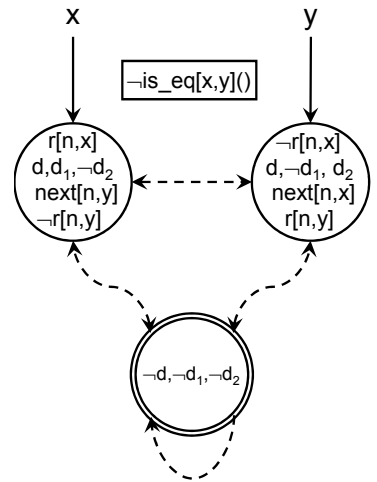

(e)

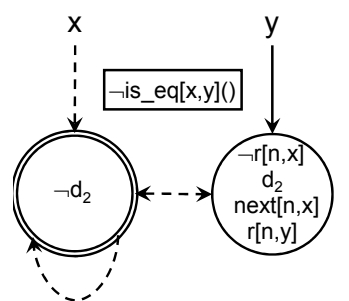

(c)

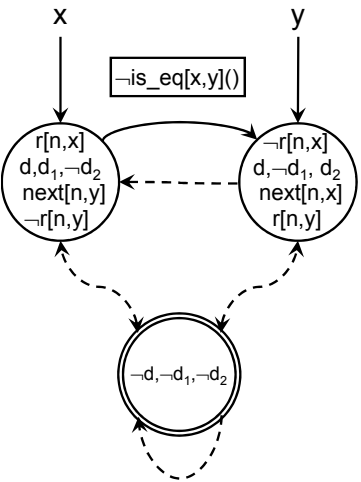

(f)

Fig. 3: Structures that arise in the meet operation used to analyze $x \mapsto y * y \mapsto x$.

at least one cell). The summary individual labeled $\neg d, \neg d_{1}, \neg d_{2}$ in Fig. 3(a) represents a region that is disjoint from $d$. (See also Fig. 6.)

Note that here and throughout the paper, for brevity the figures only show predicates that are relevant to the issue under discussion.

Meet for a Canonical-Abstraction Domain. To impose a necessary condition for $x \mapsto y * y \mapsto x$ to be satisfiable, we take the meet of $D, A_{1}$, and $A_{2}: \llbracket x \mapsto y * y \mapsto x \rrbracket \subseteq D \sqcap A_{1} \sqcap A_{2}$. Figs. 3(d), (e), and (f) show some of the structures that arise in $D \sqcap A_{1} \sqcap A_{2}$.

The meet operation in $\mathcal{A}$ is defined in terms of the greatest-lower-bound operation induced by the approximation order in the lattice B-STRUCT[Voc, $\mathbb{A}]$. Arnold et al. [1] show that in general this operation is NP-complete; however, they define an algorithm based on graph matching that typically performs well in practice $[20, \S 8.3]$. To understand some of the subtleties of meet, consider Fig. 3(d), which shows one of the structures in $D \sqcap A_{1}$ (i.e., Fig. 3(a) $\sqcap$ Fig. 3(b)).

- From the standpoint of Fig. 3(b), meet caused the summary individual labeled " $\neg d_{1}$ " to be split into two summary individuals: " $\neg d, \neg d_{1}, \neg d_{2}$ " and "d, $\neg d_{1}, d_{2} "$. 
- From the standpoint of Fig. 3(a), meet caused the summary individual labeled " $d, d_{1}, \neg d_{2}$ " to (i) become a non-summary individual, (ii) acquire the value 1 for $x, r[n, x]$, and next $[n, y]$, and (iii) acquire the value 0 for $y$ and $r[n, y]$.

Fig. 3(e) shows one of the structures in $\left(D \sqcap A_{1}\right) \sqcap A_{2}$, i.e., Fig. 3(d) $\sqcap$ Fig. 3(c), which causes further (formerly indefinite) elements to acquire definite values.

Arnold et al. develop a graph-theoretic notion of the possible correspondences among individuals in the bounded structures that are arguments to meet, and structure the meet algorithm around the set of possible correspondences [1, §4.2].

Improving Precision Using Semantic-Reduction Operators. Fig. 3(e) still contains a great deal of indefinite information because the meet operation does not take into account the integrity constraints on structures. For instance, for the structures that we use to represent states and SL statelets, we use a unary predicate $n \operatorname{ext}[n, y]$, which holds for individuals whose $n$-link points to the individual that is pointed to by $y$. This predicate has an associated integrity constraint

$$
\forall v_{1}, v_{2} . n \operatorname{ext}[n, y]\left(v_{1}\right) \wedge y\left(v_{2}\right) \Rightarrow n\left(v_{1}, v_{2}\right) .
$$

In particular, in Fig. 3(e) the individual pointed to by $x$ has next $[n, y]=1$; however, the edge to the individual pointed to by $y$ has the value $1 / 2$.

To improve the precision of the (graph-theoretic) meet, the semi-decision procedure makes use of semantic-reduction operators. The notion of semantic reduction was introduced by Cousot and Cousot [11]. Semantic-reduction operators are useful when an abstract domain is a lattice that has multiple elements that represent the same set of states. A semantic reduction operator $\rho$ maps an abstract-domain element $A$ to $\rho(A)$ such that (i) $\rho(A) \sqsubseteq A$, and (ii) $\gamma(\rho(A))=\gamma(A)$. In other words, $\rho$ maps $A$ to an element that is lower in the lattice - and hence a "better" representation of $\gamma(A)$ in $\mathcal{A}$-while preserving the meaning. In our case, the semantic-reduction operations that we use convert a set of 3-valued structures $X S$ into a "better" set of 3 -valued structures $X S^{\prime}$ that describe the same set of 2-valued structures.

A semantic-reduction operator can have two effects:

1. In some structure $S \in X S$, some tuple $p(u)$ with indefinite value $1 / 2$ may be changed to have a definite value (0 or 1$)$.

2. It may be determined that some structure $S \in X S$ is infeasible: i.e., $\gamma(S)=\emptyset$. In this case, $S$ is removed from $X S$.

The effect of a precision improvement from a type- 1 effect can cause a type- 2 effect to occur. For instance, let $u_{1}$ and $u_{2}$ be the individuals pointed to by $x$ and $y$, respectively, in Fig. 3(e).

- Fig. 3(f) is Fig. 3(e) after integrity constraint (4) has triggered a type-1 change that improves the value of $n\left(u_{1}, u_{2}\right)$ from $1 / 2$ to 1 .

- A type-2 rule can then determine that the structure shown in Fig. 3(f) is infeasible. In particular, the predicate $r[n, x](v)$ means that individual $v$ is reachable from the individual pointed to by $x$ along $n$-links. The semanticreduction rule would find that the values $x\left(u_{1}\right)=1, n\left(u_{1}, u_{2}\right)=1$, and $r[n, x]\left(u_{2}\right)=0$ represent an irreconcilable inconsistency in Fig. 3(f): the first 


$$
\begin{aligned}
& \overline{\ell \in \text { lits }, d \Vdash A_{\ell}}(\ell) \quad \frac{\varphi_{1}, d \Vdash A_{1} \quad \varphi_{2}, d \Vdash A_{2}}{\varphi_{1} \wedge \varphi_{2}, d \Vdash A_{1} \sqcap A_{2}}(\wedge) \\
& \frac{\varphi_{1}, d \Vdash S_{1} \quad \varphi_{2}, d \Vdash A_{2}}{\varphi_{1} \vee \varphi_{2}, d \Vdash A_{1} \sqcup A_{2}}(\vee) \quad \frac{\varphi_{1}, d_{1} \Vdash A_{1} \quad \varphi_{2}, d_{2} \Vdash A_{2}}{\varphi_{1} * \varphi_{2}, d \Vdash\left(\left[d=d_{1} \cdot d_{2}\right]^{\sharp} \sqcap A_{1} \sqcap A_{2}\right) \xi^{d}}(*) \\
& \frac{\varphi_{1}, d_{1} \Vdash A_{1} \quad \varphi_{2}, d_{2} \Vdash A_{2}}{\varphi_{1}-\circledast \varphi_{2}, d \Vdash\left(\left[d_{2}=d \cdot d_{1}\right]^{\sharp} \sqcap A_{1} \sqcap A_{2}\right) \xi^{d}}(-\circledast)
\end{aligned}
$$

Fig. 4: Rules for computing an abstract value that overapproximates the meaning of a formula in SL.

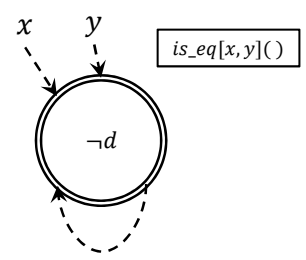

(a)

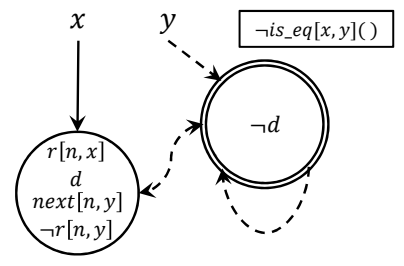

(b)

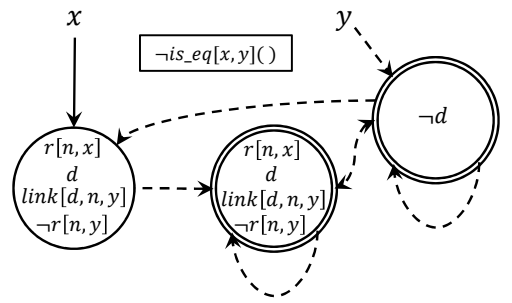

(c)

Fig. 5: The abstract value for $\mathbf{l} \mathbf{s}(x, y) \in$ atom in the canonical-abstraction domain.

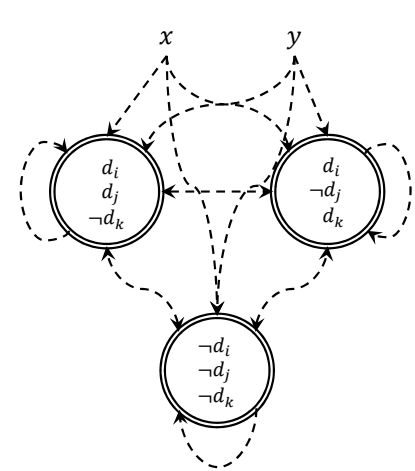

(a)

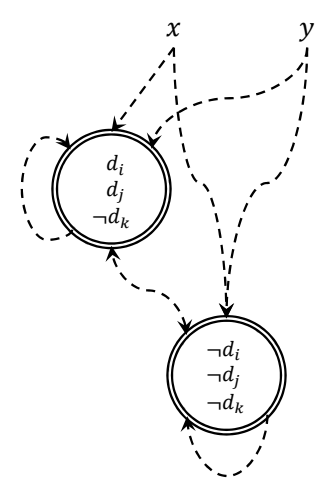

(b)

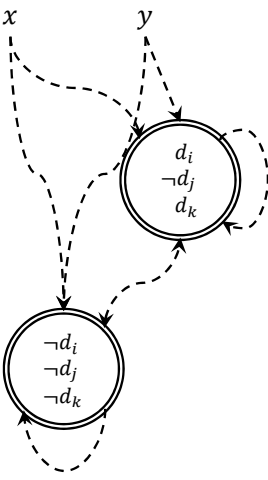

(c)

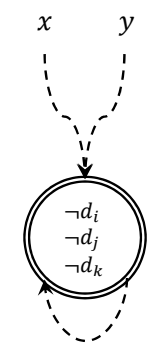

(d)

Fig. 6: The abstract value for $\left[d_{i}=d_{j} \cdot d_{k}\right]^{\sharp}$ in the canonical-abstraction domain.

two predicate values mean that $u_{2}$ is reachable from the individual pointed to by $x$ along $n$-links, which contradicts $r[n, x]\left(u_{2}\right)=0$.

The operation that applies type- 1 and type- 2 rules until no more changes are possible is called coerce (because it coerces $X S$ to a better representation $X S^{\prime}$ ). Sagiv et al. [30, §6.4] and Bogudlov et al. [5,4] discuss algorithms for coerce. 


\begin{tabular}{||l|l||}
\hline Predicate & Intended Meaning \\
\hline$i s_{-} e q[x, y]()$ & Are $x$ and $y$ equal? \\
$n e x t[n, y](v)$ & The target of the $n$-edge from $v$ is pointed to by $y$ \\
$t[n]\left(v_{1}, v_{2}\right)$ & Is $v_{2}$ reachable via zero or more $n$-edges from $v_{1} ?$ \\
$r[n, y](v)$ & $\exists v_{1} . y\left(v_{1}\right) \wedge t[n]\left(v_{1}, v\right)$ \\
$d(v)$ & Is $v$ in heap domain $d ?$ \\
$l i n k[d, n, y](v)$ & The target of the $n$-edge from $v$ is either in $d$ or is pointed to by $y$ \\
\hline
\end{tabular}

Table 1: Voc consists of the predicates shown above, together with the ones in Fig. 2. All unary predicates are abstraction predicates; that is, $\mathbb{A}=\mathrm{Voc}_{1}$.

\section{Proof System for Separation Logic}

This section describes how we compute $A \in \mathcal{A}[$ Voc, $\mathbb{A}]$ such that $A$ overapproximates the satisfying models of $\varphi \in$ SL. The vocabulary Voc and abstraction predicates $\mathbb{A}$ are listed in Tab. 1.

The semi-decision procedure works with judgments of the form " $\varphi, d \Vdash A$ ", where $d$ is a domain predicate. The invariant maintained by the semi-decision procedure is that, whenever it establishes a judgment $\varphi, d \Vdash A, A \in \mathcal{A}$ overapproximates $\varphi$ in the following sense: $\left.\gamma(A)\right|_{(d, \cdot)} \supseteq \llbracket \varphi \rrbracket$. Fig. 4 lists the rules used for calculating $\varphi, d \Vdash A$ for $\varphi \in$ SL.

For each literal $\ell \in$ lits, there is an abstract value $A_{\ell} \in \mathcal{A}$ such that $\left.\gamma\left(A_{\ell}\right)\right|_{(d, \cdot)}=\llbracket \ell \rrbracket$. These $A_{\ell}$ values are used in the $(\ell)$-rule of Fig. 4 . Fig. 5 shows the abstract value $A_{\mathbf{l s}}$ used for $\mathbf{l s}(x, y)$. $A_{\mathbf{l s}}$ consists of three structures:

- Fig. 5(a) represents the empty list from $x$ to $y$. That is, $x=y$ and region $d$ is empty.

- Fig. 5(b) represents a singleton list from $x$ to $y$. That is, $x \neq y$ and $x \neq$ nil, and for all individuals $v$ in $d, v$ is reachable from $x$ and $\operatorname{link}[d, n, y](v)$ is true. (See line 6 of Tab. 1.)

- Fig. 5(c) represents acyclic linked lists of length two or more from $x$ to $y$.

Fig. 5(b) is the single structure in $A_{x \mapsto y}$. The abstract values for atoms $x=y$, true, and emp are straightforward. We see that it is possible to represent the positive literals true, emp, $x=y, x \mapsto y$, and $\mathbf{l s}(x, y)$ precisely in $\mathcal{A}$; that is, we have $\left.\gamma A_{l}\right|_{(d .)}=\llbracket l \rrbracket$. Furthermore, because the canonical-abstraction domain $\mathcal{A}$ is closed under negation $[21,36]$, we are able to represent the negative literals $x \neq y, \neg$ true, $\neg \mathbf{e m p}, \neg \mathbf{l} \mathbf{s}(x, y)$, and $\neg x \mapsto y$ precisely in $\mathcal{A}$, as well.

The rest of the rules in Fig. 4 can be derived by reinterpreting the concrete logical operators using an appropriate abstract operator. In particular, logicaland is reinterpreted as meet, and logical-or is reinterpreted as join. Consequently, the $(\wedge)$-rule and $(\vee)$-rule are straightforward. The $(\wedge)$-rule and $(\vee)$-rule are justified by the following observation: if $\left.\gamma\left(A_{1}\right)\right|_{(d, \cdot)} \supseteq \llbracket \varphi_{1} \rrbracket$ and $\left.\gamma\left(A_{2}\right)\right|_{(d, \cdot)} \supseteq \llbracket \varphi_{2} \rrbracket$, then $\left.\gamma\left(A_{1} \sqcap A_{2}\right)\right|_{(d, \cdot)} \supseteq \llbracket \varphi_{1} \wedge \varphi_{2} \rrbracket$ and $\left.\gamma\left(A_{1} \sqcup A_{2}\right)\right|_{(d, \cdot)} \supseteq \llbracket \varphi_{1} \vee \varphi_{2} \rrbracket$.

For a given structure $A=\langle U, \iota\rangle$ and unary domain predicate $d_{i}$, we use the phrase "individuals in $d_{i}$ " to mean the set of individuals $\left\{u \in U \mid \iota\left(d_{i}\right)(u)=1\right\}$. 
The (*)-rule computes $A \in \mathcal{A}$ such that $\left.\gamma(A)\right|_{(d, \cdot)} \supseteq \llbracket \varphi_{1} * \varphi_{2} \rrbracket$. The handling of separating conjunction $\varphi_{1} * \varphi_{2}$ is based on the following insights:

- The domain predicates $d_{1}$ and $d_{2}$ are used to capture the heaplets $h_{1}$ and $h_{2}$ that satisfy $\varphi_{1}$ and $\varphi_{2}$, respectively. That is,

$$
\left.\gamma\left(A_{1}\right)\right|_{\left(d_{1}, \cdot\right)} \supseteq \llbracket \varphi_{1} \rrbracket \text { and }\left.\gamma\left(A_{2}\right)\right|_{\left(d_{2}, \cdot\right)} \supseteq \llbracket \varphi_{2} \rrbracket .
$$

$-\left[d=d_{1} \cdot d_{2}\right]^{\sharp} \in \mathcal{A}$ is used to express the constraint that the individuals in $d_{1}$ are disjoint from $d_{2}$, and that the individuals in $d$ are the disjoint union of the individuals in $d_{1}$ and $d_{2}$. With only a slight abuse of notation, the meaning of $\left[d=d_{1} \cdot d_{2}\right]^{\sharp}$ can be expressed as follows:

$$
\left.\gamma\left(\left[d=d_{1} \cdot d_{2}\right]^{\sharp}\right)\right|_{(d, \cdot)} \supseteq\left\{\left(s, h, h_{1}, h_{2}\right) \mid h_{1} \# h_{2} \text { and } h_{1} \cdot h_{2}=h\right\} .
$$

Fig. 6 shows the four structures in the abstract value $\left[d_{i}=d_{j} \cdot d_{k}\right]^{\sharp}$, where $d_{i}, d_{j}$, and $d_{k}$ are domain predicates.

$-(\cdot) \xi^{d}$ denotes the structure that results from setting the abstraction predicates to $1 / 2$ for all individuals not in $d$, and setting all domain predicates other than $d$ to $1 / 2$. In effect, this operation blurs the distinction between individuals in $d_{1}$ and $d_{2}$, and serves as an abstract method for quantifier elimination.

Using Eqns. (5) and (6) in the definition of $\varphi_{1} * \varphi_{2}$, we have

$$
\begin{aligned}
& \llbracket \varphi_{1} * \varphi_{2} \rrbracket \\
& =\left\{(s, h) \mid \exists h_{1}, h_{2} . h_{1} \# h_{2} \text { and } h_{1} \cdot h_{2}=h \text { and }\left(s, h_{1}\right) \models \varphi_{1} \text { and }\left(s, h_{2}\right) \models \varphi_{2}\right\} \\
& \subseteq \quad\left(\left[d=d_{1} \cdot d_{2}\right]^{\sharp} \quad \sqcap \quad A_{1} \quad \sqcap \quad A_{2}\right) \dot{\xi}^{d}
\end{aligned}
$$

The handling of septraction in the $(-\circledast)$-rule is similar to the handling of separating conjunction in the $(*)$-rule, except for the condition that $h_{2}=h \cdot h_{1}$. This requirement is easily handled by using $\left[d_{2}=d \cdot d_{1}\right]^{\sharp}$. App. A illustrates the application of the $(-\circledast)$-rule.

Theorem 1. The rules in Fig. 4 are sound; that is, if the rules in Fig. 4 say that $\varphi, d \Vdash A$, then $\left.\gamma(A)\right|_{(d, \cdot)} \supseteq \llbracket \varphi \rrbracket$.

The proof follows from the fact that each of the abstract operators is sound.

\section{Experimental Evaluation}

This section presents the results of our experiments to evaluate the costs and benefits of our approach. The experiments were designed to shed light on the following questions:

1. How costly is the semi-decision procedure (in terms of time)?

2. How often is the semi-decision procedure able to determine that a formula is unsatisfiable? 


\begin{tabular}{|||r||r|r||r||r|r||r|r||r||r|r|r|r||r||}
\hline \multicolumn{10}{|c|}{ Formula Characteristics } \\
\hline Formula & \multicolumn{1}{|c|}{ emp } & \multicolumn{1}{|c|}{$x=y$} & \multicolumn{1}{|c|}{$x \mapsto y$} & $\mathbf{l s}(x, y)$ & $\varphi \wedge \varphi$ & $\varphi \vee \varphi$ & $\varphi * \varphi$ & $\varphi-\circledast \varphi$ & Full \\
Group & + & - & + & - & + & - & + & - & & & & & Corpus \\
\hline \hline Group 1 & 1 & 5 & 8 & 8 & 13 & 1 & 19 & 10 & 22 & 4 & 12 & 10 & 23 \\
Group 2 & 64 & 22 & 0 & 0 & 22 & 22 & 22 & 22 & 64 & 0 & 64 & 0 & 64 \\
Group 3 & 512 & 218 & 0 & 0 & 218 & 218 & 218 & 218 & 512 & 0 & 512 & 512 & 512 \\
\hline \hline Total & 577 & 245 & 8 & 8 & 253 & 241 & 259 & 250 & 598 & 4 & 588 & 522 & 599 \\
\hline
\end{tabular}

Table 2: Number of formulas that contain each of the SL operators in the three groups of formulas used in the experiments. The columns labeled "+" and "-" indicate the number of atoms occurring as positive and negative literals, respectively.

3. For unsatisfiable formulas that are beyond the capabilities of other existing tools, is the semi-decision procedure actually able to determine that the formulas are unsatisfiable?

Setup. The semi-decision procedure is written in OCaml; it compiles a formula to a proof DAG, expressed as an equation system. The abstract-value manipulations in the proof rules of Fig. 4 are performed using ITVLA, a modified version of TVLA [23] that was implemented for performing interprocedural shape analysis $[20, \S 8]$. ITVLA (i) replaces TVLA's notion of an intraprocedural control-flow graph by the more general notion of equation system, in which transfer functions may depend on more than one argument, and (ii) supports a more general language in which to specify equation systems. In particular, the ITVLA language supports explicit use of the meet operator [1] for a canonical-abstraction domain. Experiments were run on a single core of a 2-processor, 4-core-per-processor 2.27 $\mathrm{GHz}$ Xeon computer running Red Hat Linux 6.5.

Test Suite. Our test suite consists of three groups of unsatisfiable formulas:

- Group 1, shown in Tab. 3, was chosen to evaluate our procedure on a wide spectrum of formulas.

- Group 2 was created by replacing the Boolean variables $a$ and $b$ in the template $T_{1} \stackrel{\text { def }}{=} \neg a \wedge \mathbf{e m p} \wedge(a * b)$ with the 8 literals lits of SL; that is, true, emp, $x \mapsto y, \operatorname{ls}(x, y)$, and their negations. Five of the 64 instantiations of template $T_{1}$ are shown in Tab. 4 .

- Group 3 was created by replacing the Boolean variables $a, b$, and $c$ in the template $T_{2} \stackrel{\text { def }}{=} \mathbf{e m p} \wedge a \wedge(b *(c-\circledast(\mathbf{e m p} \wedge \neg a)))$ with the 8 literals lits of SL. Five of the 512 instantiations of template $T_{2}$ are shown in Tab. 5.

Templates $T_{1}$ and $T_{2}$ are based on work by Hou et al. [19] on Boolean separation logic. Templates $T_{1}$ and $T_{2}$ are listed as formulas 15 and 19, respectively, in [19, Tab. 2]. In total, there were 599 formulas in our test suite. Tab. 2 summarizes the characteristics of the corpus based on the occurrences of the SL operators.

Though not shown in this section, we also evaluated our procedure on a set of satisfiable formulas. The procedure reports a set of abstract models when given a satisfiable formula. The time taken to compute these abstract models is similar to that for proving formulas unsatisfiable. 


\begin{tabular}{|c|c|c|c|}
\hline & Formula & Unsat & Time \\
\hline$(1)$ & $a 1 \mapsto a 2 \wedge \neg \mathbf{l}(a 1, a 2)$ & $\checkmark$ & 1.74 \\
\hline$(2)$ & $a 1 \mapsto a 2 * a 2 \mapsto a 1$ & $\checkmark$ & 1.70 \\
\hline$(3)$ & $\neg \mathbf{e m p} \wedge(\mathbf{l} \mathbf{s}(a 1, a 2) * \mathbf{l} \mathbf{s}(a 2, a 1))$ & $\checkmark$ & 2.00 \\
\hline$(4)$ & $a 1 \neq a 2 \wedge(\mathbf{l} \mathbf{s}(a 1, a 2) * \mathbf{l} \mathbf{s}(a 2, a 1))$ & $\checkmark$ & 1.97 \\
\hline$(5)$ & $(\mathbf{l} \mathbf{s}(a 1, a 2) * \mathbf{l} \mathbf{s}(a 2, a 3)) \wedge \neg \mathbf{l} \mathbf{s}(a 1, a 3)$ & $\checkmark$ & 4.46 \\
\hline$(6)$ & $\mathbf{l s}(a 1, a 2) \wedge \mathbf{e m p} \wedge a 1 \neq a 2$ & $\checkmark$ & 1.34 \\
\hline$(7)$ & $(a 1 \mapsto a 2 *$ true $) \wedge(a 2 \mapsto a 3 *$ true $) \wedge($ true $* a 3 \mapsto a 1)$ & $\checkmark$ & 4.25 \\
\hline$(8)$ & $(a 1 \mapsto a 2-\circledast$ true $) \wedge(a 1 \mapsto a 2 *$ true $)$ & $\checkmark$ & 2.43 \\
\hline$(9)$ & $(\mathbf{l} \mathbf{s}(a 1, a 2) * \neg \mathbf{l} \mathbf{s}(a 2, a 3)) \wedge \mathbf{l} \mathbf{s}(a 1, a 3)$ & $\bar{\nabla}$ & 22.2 \\
\hline$(10)$ & $\mathbf{l s}(a 1, a 2) \wedge \mathbf{l s}(a 1, a 3) \wedge \neg \mathbf{e m p} \wedge a 2 \neq a 3$ & $\checkmark$ & 1.91 \\
\hline$(11)$ & $(\operatorname{ls}(a 1, a 2) *$ true $* a 3 \mapsto a 4) \wedge(\operatorname{true} *(\mathbf{l s}(a 2, a 1) \wedge a 2 \neq a 1))$ & $\checkmark$ & 32.9 \\
\hline$(12)$ & $(a 1 \mapsto a 2 * \operatorname{ls}(e 1, e 2)) \wedge(a 2 \mapsto a 3 * \neg \mathbf{e m p}) \wedge(a 3 \mapsto a 1 * \neg a 5 \mapsto a 6 *$ true $)$ & $\checkmark$ & 39.8 \\
\hline$(13)$ & $\begin{array}{l}(\neg \mathbf{e m p} * \neg \mathbf{e m p}) \wedge(a 1=\mathrm{nil} \vee a 1 \mapsto e 1 \vee((a 1 \mapsto e 1 \wedge e 1=\mathrm{nil}) * \text { true })) \wedge \\
\mathbf{l}(a 1, a 2)\end{array}$ & $\checkmark$ & 2.34 \\
\hline$(14)$ & $\begin{array}{l}((\mathbf{l s}(a 1, a 2) \wedge a 1 \neq a 2) *(\mathbf{l s}(a 2, a 3) \wedge a 2 \neq a 3)) \wedge((\mathbf{l s}(a 4, a 1) \wedge a 4 \neq \\
a 1) * a 1 \mapsto e 1 * \text { true })\end{array}$ & $\checkmark$ & 10.8 \\
\hline$(15)$ & $(\mathbf{l s}(a 1, a 2)-\circledast \mathbf{l s}(a 1, a 2)) \wedge \neg \mathbf{e m p}$ & $\checkmark$ & 1.92 \\
\hline$(16)$ & $(a 3 \mapsto a 4-\circledast \mathbf{l}(a 1, a 4)) \wedge(a 3=a 4 \vee \neg \mathbf{l} \mathbf{s}(a 1, a 3))$ & $\checkmark$ & 2.76 \\
\hline$(17)$ & $((a 2 \mapsto a 3-\circledast \mathbf{l} \mathbf{s}(a 2, a 4))-\circledast \mathbf{l} \mathbf{s}(a 1, a 4)) \wedge \neg \mathbf{l} \mathbf{s}(a 1, a 3)$ & $\checkmark$ & 4.60 \\
\hline$(18)$ & $((a 2 \mapsto a 3-\circledast \mathbf{l} \mathbf{s}(a 2, a 4))-\circledast \mathbf{l} \mathbf{s}(a 3, a 1)) \wedge a 2=a 4$ & $\checkmark$ & 4.02 \\
\hline$(19)$ & $(a 1 \mapsto a 2-\circledast \mathbf{l} \mathbf{s}(a 1, a 3)) \wedge(\neg \mathbf{l s}(a 2, a 3) \vee($ true $\wedge(a 1 \mapsto e 1 *$ true $)) \vee a 1=a 3)$ & $\checkmark$ & 4.12 \\
\hline$(20)$ & $((\mathbf{l} \mathbf{s}(a 1, a 2) \wedge a 1 \neq a 2)-\circledast \mathbf{l} \mathbf{s}(e 1, e 2)) \wedge e 1 \neq a 1 \wedge e 2=a 2 \wedge \neg \mathbf{l}(e 1, a 1)$ & $\checkmark$ & 6.03 \\
\hline$(21)$ & $a 1 \neq a 4 \wedge(\mathbf{l} \mathbf{s}(a 1, a 4)-\circledast \mathbf{l} \mathbf{s}(e 1, e 2)) \wedge a 4=e 2 \wedge \neg \mathbf{l}(e 1, a 1)$ & $\checkmark$ & 6.92 \\
\hline$(22)$ & $((\mathbf{l} \mathbf{s}(a 1, a 2) \wedge a 1 \neq a 2)-\circledast \mathbf{l} \mathbf{s}(e 1, e 2)) \wedge e 2 \neq a 2 \wedge e 1=a 1 \wedge \neg \mathbf{l}(a 2, e 2)$ & $?$ & 6.98 \\
\hline$(23)$ & $((a 2 \mapsto a 3-\circledast \mathbf{l} \mathbf{s}(a 2, a 4))-\circledast \mathbf{l} \mathbf{s}(a 3, a 1)) \wedge(\neg \mathbf{l} \mathbf{s}(a 4, a 1) \vee a 2=a 4)$ & $?$ & 4.71 \\
\hline
\end{tabular}

Table 3: Unsatisfiable formulas.

\begin{tabular}{|l|l|l|l|}
\hline & Formula & Unsat & Time \\
\hline$(\mathbf{1})$ & $\neg(a 1 \mapsto a 2) \wedge \mathbf{e m p} \wedge(a 1 \mapsto a 2 * a 3 \mapsto a 4)$ & $\checkmark$ & 3.65 \\
\hline$(\mathbf{2})$ & $a 1 \mapsto a 2 \wedge \mathbf{e m p} \wedge(\neg(a 1 \mapsto a 2) * a 3 \mapsto a 4)$ & $\checkmark$ & 5.01 \\
\hline $\mathbf{3})$ & $\neg(a 1 \mapsto a 2) \wedge \mathbf{e m p} \wedge(a 1 \mapsto a 2 * \mathbf{l} \mathbf{s}(a 3, a 4))$ & $\checkmark$ & 6.17 \\
\hline$(\mathbf{4})$ & $\mathbf{l s}(a 1, a 2) \wedge \mathbf{e m p} \wedge(\neg \mathbf{l s}(a 1, a 2) * \mathbf{l}(a 3, a 4))$ & $\checkmark$ & 76.2 \\
\hline$(\mathbf{5})$ & $\mathbf{l s}(a 1, a 2) \wedge \mathbf{e m p} \wedge(\neg \mathbf{l s}(a 1, a 2) * \neg \mathbf{l}(a 3, a 4))$ & $\checkmark$ & 386 \\
\hline
\end{tabular}

Table 4: Example instantiations of $T_{1} \stackrel{\text { def }}{=} \neg a \wedge \mathbf{e m p} \wedge(a * b)$, where $a, b \in$ lits.

We now answer Questions 1-3 posed at the beginning of this section using the three groups of formulas.

Group 1 Results. The running time of our procedure on the formulas listed in Tab. 3 was often on the order of five seconds. The procedure was able to prove unsatisfiability for all formulas, except (22) and (23). We believe that formulas (9) $-(23)$ are beyond the scope of existing tools. Formulas (9)-(14) demonstrate that we can handle formulas that describe overlapping data structures, including conjunctions of separating conjunctions. Formulas (15)-(21) demonstrate that we can handle formulas that contain occurrences of both ls and septraction. 


\begin{tabular}{|c|c|c|c|}
\hline & Formula & Unsat & Time \\
\hline$(1)$ & $\mathbf{e m p} \wedge \mathbf{l s}(a 1, a 2) \wedge(\mathbf{l s}(a 3, a 4) *(\mathbf{l} \mathbf{s}(a 5, a 6)-\circledast(\mathbf{e m p} \wedge \neg \mathbf{l} \mathbf{s}(a 1, a 2))))$ & $\checkmark$ & 9.03 \\
\hline$(2)$ & $\mathbf{e m p} \wedge \neg \mathbf{e m p} \wedge(\mathbf{l s}(a 3, a 4) *(\neg(a 5 \mapsto a 6)-\circledast(\mathbf{e m p} \wedge \mathbf{e m p})))$ & $\checkmark$ & 3.25 \\
\hline$(3)$ & $\mathbf{e m p} \wedge a 1 \mapsto a 2 \wedge(a 3 \mapsto a 4 *(a 5 \mapsto a 6-\circledast(\mathbf{e m p} \wedge \neg(a 1 \mapsto a 2))))$ & $\checkmark$ & 3.57 \\
\hline$(4)$ & $\mathbf{e m p} \wedge \mathbf{l s}(a 1, a 2) \wedge(\neg \mathbf{l} \mathbf{s}(a 3, a 4) *(\mathbf{l s}(a 5, a 6)-\circledast(\mathbf{e m p} \wedge \neg \mathbf{l s}(a 1, a 2))))$ & $\checkmark$ & 25.3 \\
\hline$(5)$ & $\mathbf{e m p} \wedge \operatorname{ls}(a 1, a 2) \wedge(\neg \mathbf{l} \mathbf{s}(a 3, a 4) *(\neg \mathbf{l} \mathbf{s}(a 5, a 6)-\circledast(\mathbf{e m p} \wedge \neg \mathbf{l} \mathbf{s}(a 1, a 2))))$ & $\checkmark$ & 25.5 \\
\hline
\end{tabular}

Table 5: Example instantiations of $T_{2} \stackrel{\text { def }}{=} \mathbf{e m p} \wedge a \wedge(b *(c-\circledast(\mathbf{e m p} \wedge \neg a)))$, where $a, b, c \in$ lits.

Group 2 Results. The 64 formulas instantiated from the template $T_{1} \stackrel{\text { def }}{=} \neg a \wedge$ emp $\wedge(a * b)$ took between 0.82 and 386 seconds to check, with a mean of 12.4 and a median of 1.98 seconds. Our procedure was able to prove unsatisfiability for all 64 formulas. All instantiations of $T_{1}$ that contain an occurrence of the ls predicate are beyond the capabilities of existing tools.

The formulas that took the greatest amount of time and the second-greatest amount of time are (5) and (4), respectively, in Tab. 4. In both cases, a large amount of time was required because of the presence of $\neg \mathbf{l s}$, which is represented by 24 structures - a much larger number than is needed for the other literals.

Group 3 Results. The 512 formulas instantiated from the template $T_{2} \stackrel{\text { def }}{=}$ $\operatorname{emp} \wedge a \wedge(b *(c-\circledast(\operatorname{emp} \wedge \neg a)))$, took between 0.79 and 25.5 seconds to check using our procedure, with a mean of 2.98 , and a median of 2.33 seconds. Our procedure was able to prove unsatisfiability for all 512 formulas. All instantiations of $T_{2}$ that contain an occurrence of the ls predicate are beyond the capabilities of existing tools.

\section{Related Work}

The literature related to reasoning about separation logic is vast, and we mention only a small portion of it in this section. Decidability results related to first-order separation logic are discussed in $[8,6]$. A fragment of separation logic for which it is decidable to check validity of entailments was introduced in [2]. The fragment includes points-to and linked-list predicates; but no septraction, or negations of points-to or linked-list predicates. Most approaches use a syntactic proof-theoretic procedure for this fragment $[2,27]$. One exception is the approach by Cook et al. [9], which uses a more semantic approach that represents separation-logic formulas as graphs in a particular normal form and then proves that one formula entails another by computing a homomorphism between the corresponding graphs. More recent approaches deal with fragments of separation logic that are incomparable to ours $[26,22,19]$; in particular, none of these approaches handle linked lists.

The explicit use of abstract values drawn from an abstract domain as a way to represent knowledge in implementations of decision procedures is a technique that has been receiving increased attention of late $[14,33,32,15,16]$. As far as we know, our work is the first to apply this idea to a fragment of separation logic. 
Many researchers pigeonhole TVLA [23] as a system exclusively tailored for "shape analysis". In fact, it is actually a metasystem for (i) defining a family of logical structures 2-STRUCT[Voc], and (ii) defining canonical-abstraction domains whose elements represent sets of 2-STRUCT[Voc]. The ITVLA [20, $\S 8]$ variant of TVLA is a different packaging of the classes that make up the TVLA implementation, and demonstrates better that canonical abstraction is a general-purpose method for abstracting the structures that are a logic's domain of discourse.

To simplify matters, the separation-logic fragment addressed in this paper does not allow one to make assertions about numeric-valued variables and numeric-valued fields. Our approach could be extended to support such capabilities using methods developed in work on abstract interpretation that combines canonical abstraction with numeric abstractions $[18,25]$.

\section{Conclusion and Future Work}

This paper showed how to create a semi-decision procedure for a fragment of separation logic. The fragment of separation logic that we use has empty-heap assertions (emp), equalities $(x=y)$, points-to assertions $(x \mapsto y)$, acyclic-listsegment assertions $(\mathbf{l s}(x, y))$, and their negations as literals; it provides the connectives $*,-\circledast, \wedge$, and $\vee$. We believe that this is an interesting fragment, in that it contains formulas for which existing approaches do not apply.

For each SL formula $\varphi$, the procedure performs a bottom-up evaluation of the formula, using a particular shape-analysis interpretation; if the answer is the empty set of 3-valued structures, then $\varphi$ is unsatisfiable. Thus, the work reported in the paper supports the thesis that abstract-interpretation concepts can help in the design and implementation of decision procedures.

Moreover, if $\varphi$ is satisfiable, then the procedure reports a set of abstract models - i.e., a value in the canonical-abstraction domain that overapproximates $\llbracket \varphi \rrbracket$. As we have shown in other work (using a variety of other techniques, and for a variety of other logics), a decision-procedure-like method that is prepared to return such "residual" answers provides a way to generate sound abstract transformers automatically $[28,35,33,31]$. In particular, when $\varphi$ specifies the transition relation between the pre-state and post-state of a concrete transformer $\tau$, a residuating decision procedure provides a way to create a sound abstract transformer $\tau^{\sharp}$ for $\tau$, directly from a specification in logic of $\tau^{\text {'s con- }}$ crete semantics. Consequently, the work reported in the paper also supports the thesis that abstract-interpretation-based decision procedures provide much promise for automating the construction of program-analysis tools. Using our semi-decision procedure, we now have a way to create abstract transformers based on canonical-abstraction domains directly from a specification of the semantics of a language's concrete transformers, written in SL.

Although TVLA and separation logic have both been applied to the problem of analyzing programs that manipulate linked data structures, there has been only rather limited crossover of ideas between the two approaches. Our semi-decision procedure is built on the connection between TVLA states and 
SL statelets described in $\S 2.3$, which represents the first formal connection between the two approaches. For this reason, the semi-decision procedure should be of interest to both communities: (i) for the TVLA community, the procedure illustrates a different and intriguing use for canonical-abstraction domains; (ii) for the separation-logic community, the procedure shows how using TVLA and canonical-abstraction domains leads to a model-theoretic approach to the decision problem for SL that is capable of handling formulas that are beyond the capabilities of existing tools.

We believe that the approach presented in this paper has the potential to be extended to deal with richer fragments of separation logic - in particular, fragments that contain both separating implication and acyclic linked-list predicates.

\section{References}

1. G. Arnold, R. Manevich, M. Sagiv, and R. Shaham. Combining shape analyses by intersecting abstractions. In VMCAI, 2006.

2. J. Berdine, C. Calcagno, and P. O'Hearn. A decidable fragment of separation logic. In FSTTCS. 2004.

3. J. Berdine, C. Calcagno, and P. O'Hearn. Smallfoot: modular automatic assertion checking with separation logic. In FMCO, 2005.

4. I. Bogudlov, T. Lev-Ami, T. Reps, and M. Sagiv. Revamping TVLA: Making parametric shape analysis competitive. Tech. Rep. TR-2007-01-01, Tel-Aviv Univ., Tel-Aviv, Israel, 2007.

5. I. Bogudlov, T. Lev-Ami, T. Reps, and M. Sagiv. Revamping TVLA: Making parametric shape analysis competitive (tool paper). In $C A V, 2007$.

6. R. Brochenin, S. Demri, and E. Lozes. On the almighty wand. Information and Computation, 211:106-137, 2012.

7. C. Calcagno, V. Vafeiadis, and M. Parkinson. Modular safety checking for finegrained concurrency. In Static Analysis Symp., 2007.

8. C. Calcagno, H. Yang, and P. O'Hearn. Computability and complexity results for a spatial assertion language for data structures. In FSTTCS. 2001.

9. B. Cook, C. Haase, J. Ouaknine, M. Parkinson, and J. Worrell. Tractable reasoning in a fragment of separation logic. In CONCUR, 2011.

10. P. Cousot and R. Cousot. Abstract interpretation: A unified lattice model for static analysis of programs by construction or approximation of fixpoints. In POPL, 1977.

11. P. Cousot and R. Cousot. Systematic design of program analysis frameworks. In POPL, 1979.

12. D. Distefano, P. O'Hearn, and H. Yang. A local shape analysis based on separation logic. In $T A C A S, 2006$.

13. D. Distefano and M. Parkinson. jStar: towards practical verification for Java. In OOPSLA, 2008.

14. V. D'Silva, L. Haller, and D. Kroening. Satisfiability solvers are static analyzers. In $S A S, 2012$.

15. V. D'Silva, L. Haller, and D. Kroening. Abstract conflict driven learning. In Symp. on Princ. of Prog. Lang., 2013.

16. V. D'Silva, L. Haller, and D. Kroening. Abstract satisfaction. In Symp. on Princ. of Prog. Lang., 2014. 
17. K. Dudka, P. Muller, P. Peringer, and T. Vojnar. Predator: A tool for verification of low-level list manipulations. In TACAS, 2013.

18. D. Gopan, F. DiMaio, N. Dor, T. Reps, and M. Sagiv. Numeric domains with summarized dimensions. In TACAS, pages 512-529, 2004.

19. Z. Hou, R. Clouston, R. Gore, and A. Tiu. Proof search for propositional abstract separation logics via labelled sequents:. In Symp. on Princ. of Prog. Lang., 2014.

20. B. Jeannet, A. Loginov, T. Reps, and M. Sagiv. A relational approach to interprocedural shape analysis. TOPLAS, 32(2), 2010.

21. V. Kuncak and M. Rinard. On the boolean algebra of shape analysis constraints. Technical Report MIT-LCS-TR-916, M.I.T. CSAIL, Aug. 2003.

22. W. Lee and S. Park. A proof system for separation logic with magic wand. In Symp. on Princ. of Prog. Lang., 2014.

23. T. Lev-Ami and M. Sagiv. TVLA: A system for implementing static analyses. In $S A S$, pages 280-301, 2000.

24. S. Magill, J. Berdine, E. Clarke, and B. Cook. Arithmetic strengthening for shape analysis. In Static Analysis Symp., 2007.

25. B. McCloskey, T. Reps, and M. Sagiv. Statically inferring complex heap, array, and numeric invariants. In $S A S, 2010$.

26. J. Park, J. Seo, and S. Park. A theorem prover for Boolean BI. In Symp. on Princ. of Prog. Lang., 2013.

27. J. A. N. Pérez and A. Rybalchenko. Separation logic + superposition calculus $=$ heap theorem prover. In Prog. Lang. Design and Impl., 2011.

28. T. Reps, M. Sagiv, and G. Yorsh. Symbolic implementation of the best transformer. In $V M C A I, 2004$.

29. J. Reynolds. Separation logic: A logic for shared mutable data structures. In Proc. Symp. on Logic in Comp. Sci., 2002.

30. M. Sagiv, T. Reps, and R. Wilhelm. Parametric shape analysis via 3-valued logic. TOPLAS, 24(3):217-298, 2002.

31. A. Thakur, M. Elder, and T. Reps. Bilateral algorithms for symbolic abstraction. In $S A S, 2012$.

32. A. Thakur and T. Reps. A generalization of Stålmarck's method. In SAS, 2012.

33. A. Thakur and T. Reps. A method for symbolic computation of precise abstract operations. In $C A V, 2012$.

34. V. Vafeiadis and M. Parkinson. A marriage of rely/guarantee and separation logic. In CONCUR, 2007.

35. G. Yorsh, T. Reps, and M. Sagiv. Symbolically computing most-precise abstract operations for shape analysis. In TACAS, 2004.

36. G. Yorsh, T. Reps, M. Sagiv, and R. Wilhelm. Logical characterizations of heap abstractions. ACM Trans. Comput. Log., 8(1), 2007. 


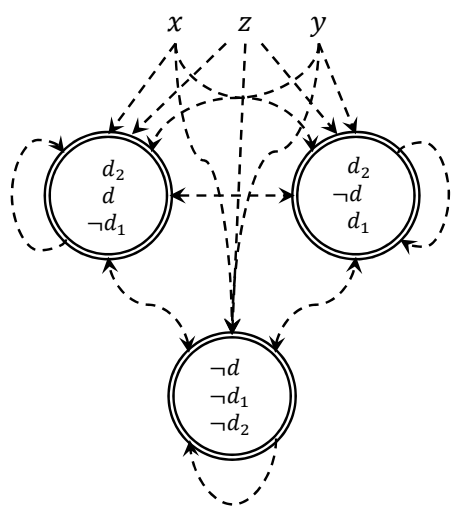

(a)

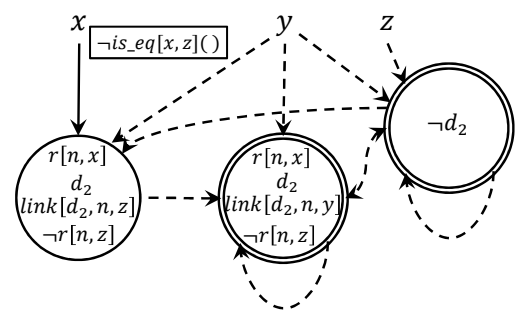

(c)

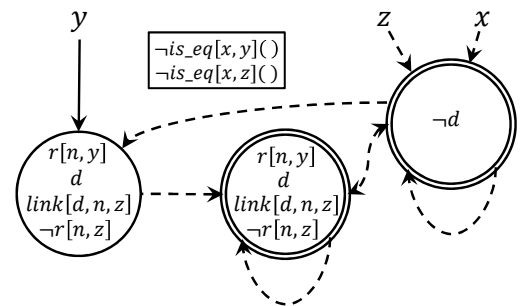

(e)

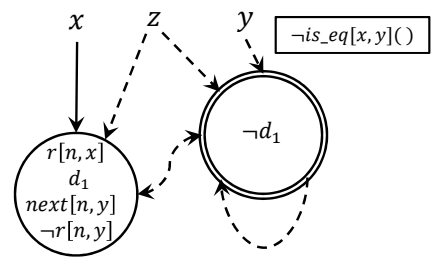

(b)

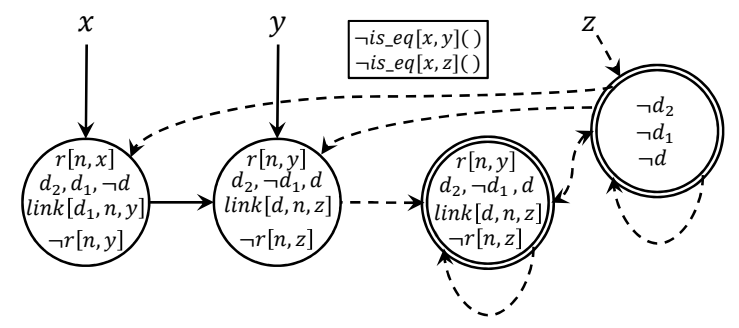

(d)

Fig. 7: Some of the structures that arise in the meet operation used to evaluate $x \mapsto y-\circledast \mathbf{l}(x, z)$.

\section{A A Satisfiable Formula}

Consider the formula $\varphi \stackrel{\text { def }}{=} x \mapsto y-\circledast \mathbf{l} \mathbf{s}(x, z)$. We want to compute $A \in \mathcal{A}$ such that $\left.\gamma(A)\right|_{(d, \cdot)} \supseteq \llbracket \varphi \rrbracket$. Similar to what was done in $\S 3$ for the $*$ operator, we introduce two new domain predicates $d_{1}$ and $d_{2}$, which are used to demarcate the heaplets that must satisfy $\varphi_{1} \stackrel{\text { def }}{=} x \mapsto y$ and $\varphi_{2} \stackrel{\text { def }}{=} \mathbf{l}(x, z)$. By design, there exist $A_{1}, A_{2} \in \mathcal{A}$ such that $\left.\gamma\left(A_{1}\right)\right|_{\left(d_{1}, \cdot\right)}=\llbracket x \mapsto y \rrbracket$ and $\left.\gamma\left(A_{2}\right)\right|_{\left(d_{2}, \cdot\right)}=\llbracket \mathbf{l} \mathbf{s}(x, z) \rrbracket$, respectively. $A_{1}$ consists of the single 3 -valued structure shown in Fig. $7(\mathrm{~b})$. Fig. 7(c) shows one of the structures in $A_{2}$; it represents an acyclic linked list

from $x$ to $z$ whose length is greater than 1 . Furthermore, to satisfy $\varphi_{1}-\circledast \varphi_{2}, d$ and $d_{1}$ are required to be disjoint regions whose union is $d_{2}$. $\mathcal{A}$ also contains an 
abstract value, which we will call $D$, that represents this disjointness constraint exactly. $D$ consists of four 3 -valued structures. Fig. 7 (a) shows the "most general" of them: it represents two disjoint regions, $d$ and $d_{1}$, that partition the $d_{2}$ region (where each of $d$ and $d_{1}$ contain at least one cell). The summary individual labeled $\neg d, \neg d_{1}, \neg d_{2}$ in Fig. 7 (a) represents a region that is disjoint from $d_{2}$.

To impose a necessary condition for $x \mapsto y-\circledast \mathbf{l} \mathbf{s}(x, z)$ to be satisfiable, we take the meet of $D, A_{1}$, and $A_{2}: \llbracket x \mapsto y-\circledast \mathbf{l s}(x, z) \rrbracket \subseteq D \sqcap A_{1} \sqcap A_{2}$. Fig. 7(d) shows one of the structures that arises in $D \sqcap A_{1} \sqcap A_{2}$, after the semantic-reduction operators have been applied. A few points to note about this resultant structure:

- The summary individual in region $d_{2}$ present in the $\mathbf{l s}(x, z)$ structure in Fig. 7(c) is split in Fig. 7(d) into a singleton individual pointed to by $y$ and a summary individual.

- The individual pointed to by $x$ is in regions $d_{1}$ and $d_{2}$, but not $d$.

- The individual pointed to by $y$ is in regions $d$ and $d_{2}$, but not $d_{1}$.

- The variables $x$ and $y$ are not equal.

- All the individuals in $d$ are reachable from $y$, not reachable from $z$, and have link $[d, n, z]$ true.

Fig. 7(e) shows the structure after we have projected the heap onto the heap region $d$; that is, the values of the domain predicates $d_{1}$ and $d_{2}$ have been set of $1 / 2$ on all individuals, and all the abstraction predicates have been set to $1 / 2$ on all individuals not in $d$. In effect, this operation blurs the distinction between the region that is outside $d$, but in $d_{2}$, and the region that is outside of $d$ and $d_{2}$. Note that the fact that $x$ and $y$ are not equal is preserved by the projection operation. This projection operation, denoted by $(\cdot)^{d}$ in $\S 4$, serves as an abstract method for quantifier elimination.

Note that Fig. 7(e) represents an acyclic linked-list from $y$ to $z$ with $x \neq y$, which is one of the models that satisfies $x \mapsto y-\circledast \mathbf{l}(x, z)$. 